\title{
Formas especiales de injuria renal aguda (IRA) en Latinoamérica: IRA secundaria al uso de herbolaria
}

\author{
Especial forms of acute kidney injury (AKI) in Latin America: AKI induced by the use of \\ herbal products
}

\author{
Rolando Claure-Del Granado ${ }^{1,2 *}$ y Alejandra Pérez-Irigoyen ${ }^{3}$ \\ ${ }^{1}$ Servicio de Nefrología, Hospital Obrero N² 2, C.N.S., Cochabamba, Bolivia; ${ }^{2}$ Universidad Mayor de San Simón, Cochabamba, Bolivia; ${ }^{3}$ Universidad \\ Iberoamericana, Ciudad de México, México
}

\section{Introducción}

La Real Academia Española define la herbolaría como la botánica aplicada a la medicina. La medicina herbolaria se define como un producto derivado de una planta, el cual es utilizado con fines terapéuticos. Esta medicina incluye un gran número de sustancias extraídas de hierbas que van desde el té hecho en casa hasta productos que tienen que ser aprobados por organismos de regulación gubernamental ${ }^{1}$. En muchos lugares, basándose en el argumento de que «lo natural es lo más seguro", en las dos últimas décadas, y a pesar de brindarse suficiente atención a la garantía y control de calidad de estos medicamentos, su uso va en aumento y desafortunadamente, de la misma manera, el número de pacientes que han sufrido daños a la salud ocasionados por su consumo se ha incrementado ${ }^{2,3}$.

En países con economías emergentes, hasta el $80 \%$ de su población depende de los sistemas de medicina herbolaria como su fuente primaria de salud. Por otro lado, en la Comunidad Europea, la medicina herbolaria representa una importante proporción del mercado farmacéutico ${ }^{4}$. En diferentes países de América Latina y el Caribe, la medicina tradicional representa una opción importante de respuesta ante las necesidades de atención a la salud ${ }^{4}$. En China, el $40 \%$ de la atención sanitara está a cargo de la medicina tradicional ${ }^{4}$.

Entre el gran número de enfermedades crónicas tratadas con medicina herbolaria podemos encontrar las enfermedades renales. El efecto secundario de los medicamentos inmunosupresores, como los corticoesteroides empleados para el tratamiento de las enfermedades glomerulares, y la falta de acceso a estas opciones terapéuticas en muchos países, especialmente para las personas con ingresos económicos medio-bajos, ha llevado a muchos pacientes a la búsqueda de una alternativa terapéutica. Sin embargo, se sabe que muchas plantas medicinales causan nefrotoxicidad. Este efecto adverso de la medicina herbolaria, por lo general, no lo toma en cuenta la mayoría de los pacientes que la utilizan debido a la creencia errónea de que los medicamentos a base de hierbas son inocuos 5 .

\section{La medicina herbolaria como causa de injuria renal aguda}

La causa más frecuente de injuria renal aguda (IRA) secundaria a nefrotóxicos en el sur de África y Asia es el uso de hierbas medicinales; por ejemplo, el empleo de hierbas medicinales tradicionales ha sido asociado al $37 \%$ de todos los casos de IRA en Nigeria ${ }^{6}$. Existen

\footnotetext{
Correspondencia:

Fecha de recepción: 25-08-2018

Disponible en internet: 19-10-2018 
varias plantas medicinales asociadas a daño renal, entre las más frecuentes están la Securidaca longepedunculata (árbol violeta), que contiene metil-salicilato y saponina; Euphorbia matabelensis, que contiene látex; Crotalaria laburnifolia, que contiene alcaloides hepato y nefrotóxicos; Uncaria tomentosa (uña de gato); Lepidium meyenii (maca); Tripterygium wilfordii (Lei Gong Teng); raíz de licoride (Glycyrrhiza glabra); Callilepis laureola (Impila); y el Cape aloes (aloe vera o sábila). A continuación, se describen ejemplos importantes de la nefrotoxicidad causada por herbolaria o medicina tradicional.

En China se usa desde hace más de 2000 años la hierba T. wilfordii Hook F (TWHF). Como crema, se usa de manera tópica para el tratamiento de la artritis y diversos procesos inflamatorios agudos. Como extracto, esta hierba es administrada por vía oral para el tratamiento de la artritis reumatoide, lupus, enfermedad de cambios mínimos y otras enfermedades autoinmunes. Los efectos adversos de esta hierba incluyen: alteraciones gastrointestinales, infertilidad, e in vitro se ha demostrado inhibición de la proliferación de linfocitos. A nivel renal, en ratas, la ingestión diaria de uno de los compuestos de la TWHF durante 16 días condicionó el desarrollo de necrosis tubular aguda en estos animales?

En Perú, la uña de gato es una preparación de herbolaria hecha de la Uncaria, una vid leñosa que se encuentra en los lechos del Amazonas. La uña de gato ha sido empleada para el tratamiento de la cirrosis, gastritis, gonorrea, neoplasias del aparato genito-urinario y reumatismo. Se cree que los alcaloides de oxindol de la corteza de la raíz son los que proveen efectos medicinales en contra de procesos inflamatorios; pero, además, en esta vid existen otras sustancias no conocidas que contribuyen al efecto global del extracto de uña de gato. En relación con posibles efectos adversos a nivel renal se han reportado casos de nefritis intersticial aguda tras el uso de uña de gato, como una reacción alérgica idiosincrática al uso de esta medicina tradicional ${ }^{8}$.

Finalmente, en relación con el uso de hongos e IRA, la mayor parte de los casos han sido consecuencia de IRA secundaria a falla hepática inducida por hongos. Sin embargo, en Europa y Norteamérica existe una variedad de hongos potencialmente nefrotóxicos que pueden ser confundidos con hongos comestibles y que causan IRA directamente. Las especies de Cortinarius (calliteus, cinnamomeus, gentilis, orellanus, rainierensis, speciosissimus, splendens, semisanguineus) son los más notables. El hongo nefrotóxico más común, probablemente, es el C. gentilis. En 2011 se reportaron cerca de 6,818 exposiciones a este hongo en EE.UU., Samoa americana, los Estados Federados de
Micronesia, Guam, Puerto Rico y las Islas Vírgenes estadounidenses, con dos casos fatales, pero solo ocho casos fueron atribuidos al grupo de Cortinarius ${ }^{9}$. Sin embargo, en más del $80 \%$ de los casos se desconoce el tipo de hongo al cual estuvieron expuestos los pacientes, por lo que es muy probable que la exposición a especies de Cortinarius sea mayor. En relación con los síntomas, los gastrointestinales, generalmente, se encuentran presentes inmediatamente tras la ingestión, y por lo general son leves, lo que evita que los pacientes busquen atención médica inmediatamente; los síntomas de IRA, generalmente, no se presentan hasta 1 a 3 semanas tras la exposición. Un menor tiempo de latencia sugiere mayor toxicidad y un mayor riesgo de IRA grave. La mejoría de la función renal puede tomar de varias semanas a meses, pero algunos pacientes evolucionan a enfermedad renal crónica terminal, por lo que requieren terapia de soporte renal crónica o trasplante.

Recientemente se han descrito casos de necrosis tubular aguda asociada a otras especies de hongos como Amanita smithiana o Amanita próxima. Se cree que la toxina que ocasiona el daño renal es el ácido 2-amino-4,5 hexadecoico ${ }^{10}$. A pesar de ocasionar necrosis tubular aguda en horas después de la ingestión de estos hongos, la evolución clínica es por lo general buena, con recuperación total de la función renal previa.

\section{¿Cuál es el problema en nuestra región?}

En Latinoamérica existen alrededor de 95,000 especies de plantas. Esta gran biodiversidad ha sido y continúa siendo una importante fuente de ingresos económicos, y es parte de la cultura, de la religión y del cuidado de la salud ${ }^{11}$. Las prácticas de medicina tradicional en Latinoamérica son muy variadas y diferentes en cada región y están en estrecha relación con la gran biodiversidad existente. La medicina tradicional constituye una parte importante del sistema de salud de muchas poblaciones indígenas en nuestra región $y$, por ejemplo, la medicina tradicional incluso ha sido integrada al sistema nacional de salud en Bolivia. La medicina tradicional es en muchos sitios la medicina de primer contacto o única disponible para pacientes en áreas rurales y para algunos en áreas urbanas ${ }^{12-15}$.

También debemos mencionar que mucha de la medicina tradicional que proviene de la flora local ha sido estudiada y desarrollada en medicinas tradicionales muy conocidas. La quinina derivada de la corteza del árbol de la Cinchona fue empleada para el tratamiento de la fiebre antes de ser desarrollada en 
el medicamento para tratar la malaria. De manera similar, la ipecacuana (Cephaelis ipecacuanha) fue usada previamente para la dispepsia, el asma y la disentería amebiana; finalmente el curare (Chondrodendron tomentosum) históricamente se ha empleado para envenenar flechas utilizadas en la caza ${ }^{16}$.

Miles de plantas nativas tienen efectos biológicos que han permitido su uso durante siglos; sin embargo, la mayor parte del conocimiento sobre sus efectos ha sido y continúa siendo transmitido oralmente de generación en generación; por lo que se conoce muy poco acerca de los tipos, razones, formas de administración, beneficios y efectos adversos de la medicina tradicional en nuestra región ${ }^{11}$. Incluso en Perú, donde el Centro Nacional de Salud Intercultural trabaja para catalogar la medicina tradicional en el país, la mayor parte de la medicina tradicional continua sin ser identificada y muy poca ha sido estudiada en relación con la evidencia de beneficios terapéuticos o su seguridad ${ }^{17}$.

La prevalencia de gente que busca a la medicina tradicional para tratar síntomas o problemas de salud varía ampliamente de país a país, y es de un $40 \%$ en Colombia y un $70 \%$ en Chile, pero tomando en cuenta los escasos datos sobre el uso de medicina tradicional en Latinoamérica quizás estos números infra estimen la importancia de la medicina tradicional en muchas poblaciones de nuestra región ${ }^{14}$.

En nuestra región, la mayor parte de los casos de IRA están relacionados con la uña de gato, ya descrita anteriormente ${ }^{8,18}$. En la región de los Andes, una hierba denominada yacón (Smallanthus sonchifolius), usada frecuentemente para tratar la diabetes debido a sus efectos hipoglucemiantes, ha sido asociada en modelos animales a nefrotoxicidad tras exposición prolongada ${ }^{19}$. La sábila 0 aloe vera (Cape aloes) ha sido introducida en el mercado regional. En Bolivia, por ejemplo, existe un boom sobre sus beneficios y usos, especialmente en pacientes con diversos tipos de neoplasias. Existen diversas presentaciones comerciales, desde preparaciones puras hasta agua mezclada con aloe (Figs. 1 y 2), lo que ha ocasionado un incremento en su consumo y reportes de IRA asociada a su consumo en pacientes con factores de riesgo. La maca, también mencionada con anterioridad y que es una causa frecuente de necrosis tubular aguda en África, es usada en Bolivia como un afrodisiaco, energizante, y es conocido localmente como «el Viagra Andino». Lamentablemente este producto incluso es comercializado por laboratorios locales y cuenta incluso con registro sanitario (Fig. 3). Finalmente, en América Central, la prevalencia de enfermedad renal de origen desconocido

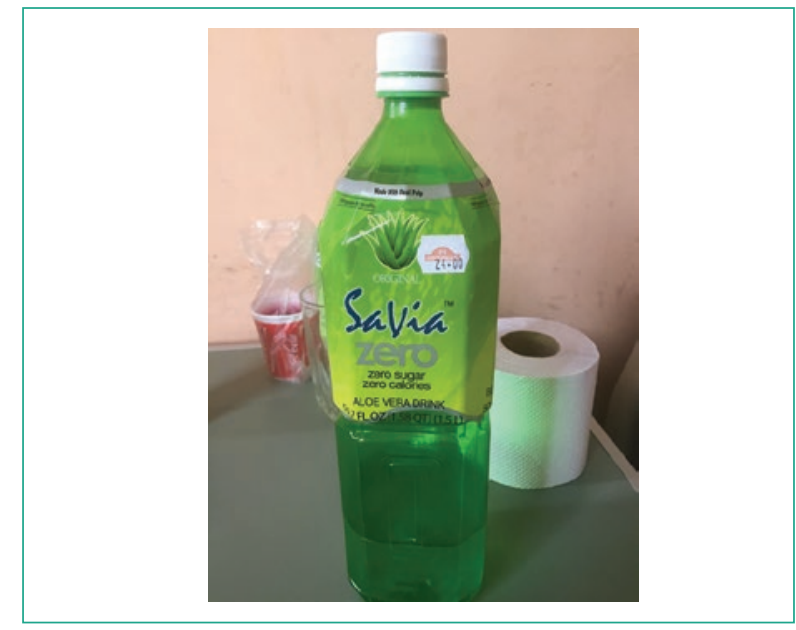

Figura 1. Bebida de agua más aloe vera comercializada en Bolivia en varios supermercados.

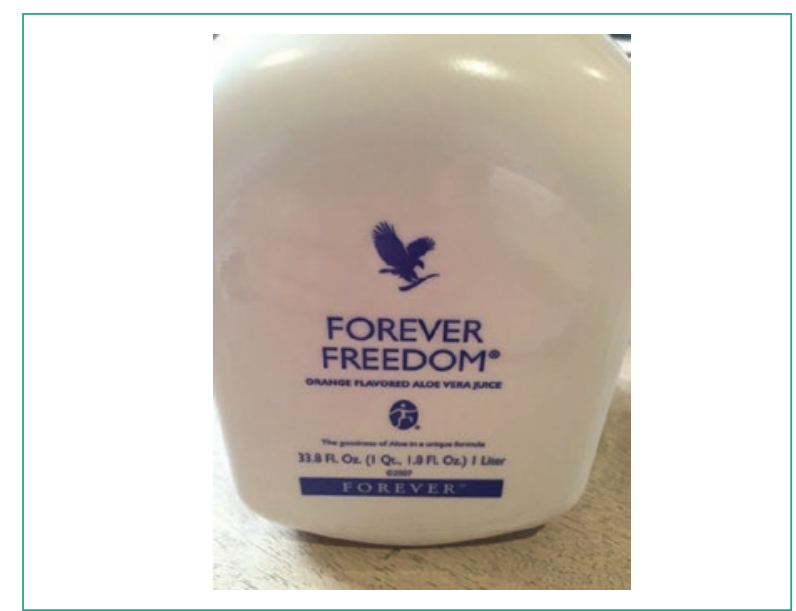

Figura 2. Bebida proveniente de EE.UU. comercializada en Bolivia que contiene aloe vera.

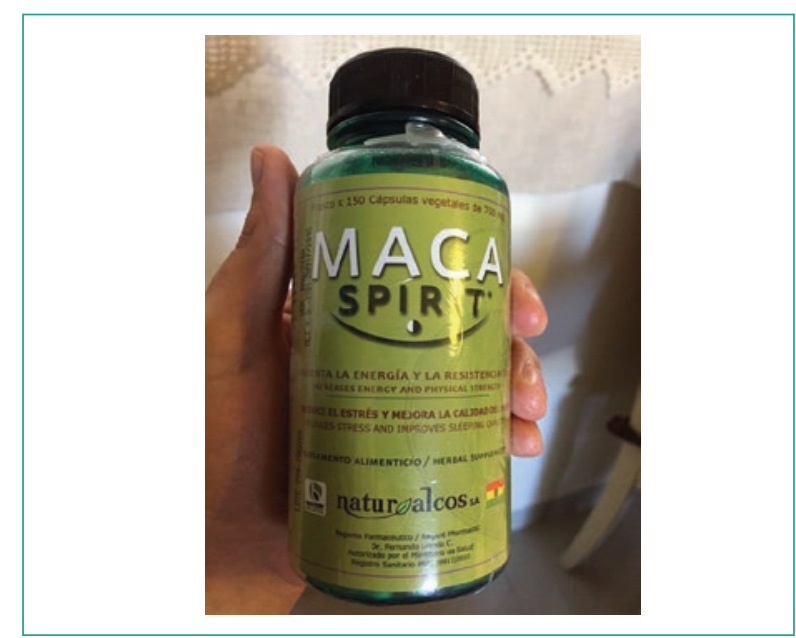

Figura 3. Medicamento producido por laboratorios locales de Bolivia que contiene Maca. 
Tabla 1. Sitios Web que proporcionan datos sobre riesgos del empleo de herbolaria

Sitio web
www.fda.gov
www.nnlm.nlm.nih.gov/pnr/uwmhg/
www.nlm.nih.gov/medlineplus/herbalmedicine.html
www.amfoundation.org
www.herbmed.org
http://nccam.nih.gov
http:// http://toxnet.nlm.nih.gov nccam.nih.gov

o nefropatía mesoamericana (ERCd) ha crecido tanto que, en el Salvador, la enfermedad renal crónica terminal secundaria a ERCd es la principal causa de muer$t^{20}$. Muchas etiologías han sido especuladas, pero la medicina tradicional podría tener un potencial rol en su desarrollo el cual no ha sido estudiado ${ }^{21}$.

\section{Conclusiones}

Diversas enfermedades renales pueden ser ocasionadas o empeoradas por el uso de medicina tradicional, dependiendo de varios factores como: características propias del paciente, tipo de herbolaria empleada, modo de administración y frecuencia de su uso. Debido al poco conocimiento que se tiene sobre los efectos adversos del uso de mucha de la medicina tradicional, los nefrólogos e investigadores deben, en coordinación con los servicios de salud pública, implementar estrategias efectivas para reconocer las diferentes medicinas tradicionales que se emplean e investigar cuidadosamente los diferentes efectos adversos, o potencialmente beneficiosos asociados a su uso. Finalmente se requiere crear conciencia en la población y en el personal de salud, a través por ejemplo del uso de sitios web que contienen datos sobre los riesgos del empleo de herbolaria o medicina tradicional (Tabla 1), sobre los efectos adversos de la medicina tradicional en poblaciones con riesgo para enfermedad renal crónica o IRA.

\section{Bibliografía}

1. Vickers A, Zollman C, Lee R. Herbal medicine. West J Med. 2001;175(2): 125-8.

2. The Efficacy and Safety of Medicinal Herbs International Conference. 2-3 March 2000, Chapel Hill, North Carolina, USA. Public Health Nutr. 2000;3(4A):445-535.

3. Arab L. Medicinal herbs: naturally safe. Public Health Nutr. 2000;3(2):111.

4. Arab L. Epidemiologic challenges in the study of the efficacy and safety of medicinal herbs. Public Health Nutr. 2000;3(4A):453-7.

5. Lin MY, Chiu YW, Chang JS, Lin HL, Lee CT, Chiu GF, et al. Association of prescribed Chinese herbal medicine use with risk of end-stage renal disease in patients with chronic kidney disease. Kidney Int 2015;88(6):1365-73.

6. Kadiri S, Ogunlesi A, Osinfade K, Akinkugbe OO. The causes and course of acute tubular necrosis in Nigerians. Afr J Med Med Sci. 1992:21(1):91-6.

7. Dan H, Peng RX, Ao Y, Liu YH. Segment-specific proximal tubule injury in tripterygium glycosides intoxicated rats. J Biochem Mol Toxicol. 2008;22(6):422-8.

8. Hilepo JN, Bellucci AG, Mossey RT. Acute renal failure caused by 'cat's claw' herbal remedy in a patient with systemic lupus erythematosus. Nephron. 1997;77(3):361.

9. Bronstein AC Spyker DA, Cantilena LR, Jr, Rumack BH, Dart RC 2011 Annual report of the American Association of Poison Control Centers' National Poison Data System (NPDS): $29^{\text {th }}$ Annual Report. Clin Toxicol (Phila). 2012:50(10):911-1164.

10. Saviuc $P$, Danel V. New syndromes in mushroom poisoning. Toxicol Rev. 2006;25(3):199-209.

11. Growing threat from counterfeit medicines. Bull World Health Organ. 2010;88(4):247-8.

12. Pedersen $D$, Baruffati $V$. Health and traditional medicine cultures in Latin America and the Caribbean. Soc Sci Med. 1985;21(1):5-12.

13. Miranda JJ, Nunez H, Alca A. Traditional healers, still part of the community health systems in the Andes. J Epidemiol Community Health. 2002;56(10):733.

14. Montenegro RA, Stephens $C$. Indigenous health in Latin America and the Caribbean. Lancet. 2006;367(9525):1859-69.

15. Anderson I, Robson B, Connolly M, Al-Yaman F, Bjertness E, King A, et al. Indigenous and tribal peoples, health (The Lancet-Lowitja Institute Global Collaboration): a population study. Lancet. 2016;388(10040):131-57.

16. Gonzalez-Coloma A, Reina M, Saenz C, Lacret R, Ruiz-Mesia L, Aran VJ, et al. Antileishmanial, antitrypanosomal, and cytotoxic screening of ethnopharmacologically selected Peruvian plants. Parasitol Res. 2012;110(4):1381-92.

17. Catalogue Florístico De Plantas Medicinales Peruanas-Lima: Centro Nacional De Salud Intercultural, Instituto Nacional De Salud. 2013. Disponible en: http://www.bvs.ins.gob.pe/insprint/CENSI/catalogo_floristico_ plan tas_medicinales.pdf.

18. Valerio LG, Jr., Gonzales GF. Toxicological aspects of the South American herbs cat's claw (Uncaria tomentosa) and Maca (Lepidium meyenii): a critical synopsis. Toxicol Rev. 2005;24(1):11-35.

19. De Oliveira RB, de Paula DA, Rocha BA, Franco JJ, Gobbo-Neto L, Uyemura SA, et al. Renal toxicity caused by oral use of medicinal plants: the yacon example. J Ethnopharmacol. 2011;133(2):434-41.

20. Rodriguez MI. Sounding the alarm on chronic kidney disease in farming communities: Maria Isabel Rodriguez MD. Minister of health, El Salvador. Interview by Conner Gorry. MEDICC Rev. 2013;15(3):8-10.

21. Lunyera J, Mohottige D, Von Isenburg M, Jeuland M, Patel UD, Stanifer JW. CKD of Uncertain Etiology: A Systematic Review. Clin J Am Soc Nephrol. 2016;11(3):379-85. 